\title{
STABILITY ESTIMATE FOR A STRONGLY COUPLED PARABOLIC SYSTEM
}

\author{
JISHAN FAN AND KUN-CHU CHEN*
}

\begin{abstract}
We consider an inverse source problem for a $2 \times 2$ strongly coupled parabolic system. The Lipschitz stability is proved and the proof is based on the Carleman estimates with two large parameters.
\end{abstract}

\section{Introduction}

We consider the following strongly coupled parabolic system

$$
\left\{\begin{array}{l}
\partial_{t} u_{1}=a_{11}(x, t) \Delta u_{1}+a_{12}(x, t) \Delta u_{2}+\widetilde{\ell}_{1}\left(u_{1}, \nabla u_{1}, u_{2}, \nabla u_{2}\right)+\widetilde{f}_{1}(x, t), \\
\partial_{t} u_{2}=a_{21}(x, t) \Delta u_{1}+a_{22}(x, t) \Delta u_{2}+\widetilde{\ell}_{2}\left(u_{1}, \nabla u_{1}, u_{2}, \nabla u_{2}\right)+\widetilde{f}_{2}(x, t)
\end{array}\right.
$$

in $Q:=\Omega \times(0, T)$ with initial and boundary conditions

$$
\left\{\begin{array}{l}
\left.\left(u_{1}, u_{2}\right)\right|_{\partial \Omega \times(0, T)}=0, \\
\left.\left(u_{1}, u_{2}\right)\right|_{t=0}=\left(u_{01}(x), u_{02}(x)\right) \text { in } \Omega \subseteq \mathbb{R}^{n} .
\end{array}\right.
$$

Here $\Omega \subseteq \mathbb{R}^{n}$ is a bounded domain with smooth boundary $\partial \Omega . A(x, t):=\left(a_{i j}\right)_{2 \times 2}$ is a smooth positive matrix with different eigenvalues. $\widetilde{\ell}_{i}(i=1,2)$ are smooth linear functions and $\widetilde{f}_{i}(i=$ $1,2)$ are smooth unknown functions. It can be proved (see Appendix) that there exists an invertible matrix $P(x, t)=\left(p_{i j}\right)_{2 \times 2}$ such that

$$
P^{-1} A P=\left(\begin{array}{cc}
\mu_{1} & 0 \\
\mu_{0} & \mu_{2}
\end{array}\right)
$$

with $\mu_{1}>0, \mu_{2}>0$. Let

$$
\left(\begin{array}{l}
u \\
v
\end{array}\right)=P^{-1}\left(\begin{array}{l}
u_{1} \\
u_{2}
\end{array}\right),\left(\begin{array}{c}
f_{1} \\
f_{2}
\end{array}\right)=P^{-1}\left(\begin{array}{c}
\widetilde{f}_{1} \\
\widetilde{f}_{2}
\end{array}\right)
$$

Corresponding author: .

2010 Mathematics Subject Classification. 35Q30, 35R30.

Key words and phrases. Inverse source problem, Lipschitz stability, Carleman estimates.

* Partially supported by the National Science Council and NCTS of Taiwan. 
and similarly, we denote $\ell_{i}(u, \nabla u, v, \nabla v)(i=1,2)$ the linear function of $u, \nabla u, v, \nabla v$ with the coefficients depending on $(x, t)$. Therefore, it is natural to consider the following system

$$
\left\{\begin{array}{l}
\partial_{t} u=\mu_{1} \Delta u+\ell_{1}(u, \nabla u, v, \nabla v)+f_{1}(x, t), \\
\partial_{t} v=\mu_{0} \Delta u+\mu_{2} \Delta v+\ell_{2}(u, \nabla u, v, \nabla v)+f_{2}(x, t), \\
u=v=0 \text { on } \partial \Omega \times(0, T) .
\end{array}\right.
$$

Our inverse problem is to find $\left\{f_{1}(x, t), f_{2}(x, t)\right\}$ from the input data

$$
\left.(u, v)\right|_{\omega \times(0, T)} \text { and }(u, v)\left(x, t_{0}\right), x \in \Omega, t_{0} \in(0, T) .
$$

Here $\omega \subset \subset \Omega$ is a given subdomain. Our aim is to prove the Lipschitz stability of the inverse source problem. The key ingredient is a Carleman estimate with two large parameters due to Yamamoto [1]. The pioneering paper for the inverse problem by Carleman estimate was obtained by Bukhgeim and Klibanov [2]. For the related work, Klibanov [3] got estimates of initial conditions of parabolic equations and inequalities via lateral cauchy data. The first Lipschitz stability result of an inverse source problem for a parabolic equation was obtained by Imanuvilov and Yamamoto [4]. Moreover the method of [4] was generalized to the inverse source problem of Navier-Stokes equation [5] and the Boussinesq system [6], respectively. Very recently, Benabdallah-Cristofol-Gaitan-Yamamoto [7] study our inverse problem (1.1) with $\mu_{0}=0$. We note that the method of [7] can not be adapted to our system.

We make the following assumptions.

$$
\left\{\begin{array}{l}
(H 1)\left|\partial_{t} f_{i}(x, t)\right| \leq c\left|f_{i}\left(x, t_{0}\right)\right|(i=1,2) \quad(x, t) \in \bar{\Omega} \times[0, T] . \\
(H 2) \mu_{0}, \mu_{1}, \mu_{2} \in C^{2}(\bar{\Omega} \times[0, T]) \quad \mu_{1}>0, \mu_{2}>0 .
\end{array}\right.
$$

Each $\ell_{i}(u, \nabla u, v, \nabla v)$ denotes a first order different differential operator acting on $u$ and on $v$ with $C^{2}$ coefficients depending on $(x, t)$.

Remark 1.1. (H1) is also assumed in [4]. Therefore, there exists a positive constant $c$ such that

$$
\left|f_{i}(x, t)\right|+\left|\partial_{t} f_{i}(x, t)\right| \leq c\left|f_{i}\left(x, t_{0}\right)\right|(i=1,2)
$$

for $(x, t) \in \bar{\Omega} \times[0, T]$. In [4], $f_{i}(x, t)=p_{i}(x) R_{i}(x, t)$ where $R_{i}(x, t)$ is given, and $R_{i}\left(x, t_{0}\right) \neq 0$. Then we are in a position to state our main result in this paper.

Theorem 1.1. Let (H1) and (H2) be satisfied. Then there exists a positive constant $c:=c\left(\Omega, T, t_{0}\right)$ such that

$$
\left\|f_{1}\right\|_{L^{2}(Q)}+\left\|f_{2}\right\|_{L^{2}(Q)} \leq c\left\{\|u(\cdot, T / 2)\|_{H^{2}}+\|v(\cdot, T / 2)\|_{H^{2}}+\|u\|_{H^{1}\left(0, T ; L^{2}(\omega)\right)}+\|v\|_{H^{1}\left(0, T ; L^{2}(\omega)\right)}\right\} .
$$


Remark 1.2. If $t_{0}=0$, then the corresponding inverse problem for a parabolic equation is open (cf Isakov $[8,9]$ ); our inverse problem with $t_{0}=0$ is also open.

Remark 1.3. We can not prove a similar result for an $m \times n$ strongly coupled parabolic system for $m, n \geq 3$. It is an open problem posed in [1] to prove a Carleman estimate for $n \times n$ strongly coupled parabolic system for $n \geq 2$. Thus we solve the open problem when $n=2$.

\section{Key Carleman estimate}

For our Carleman estimate we need a weight function with special properties. The existence of such a function is proved in $[10,11]$.

Lemma 2.1. Let $\omega_{0}$ be an arbitrary fixed sub-domain of $\Omega$ such that $\bar{\omega}_{0} \subset \omega$. Then there exists a function $\eta \in C^{2}(\bar{\Omega})$ such that

$$
\eta(x)>0 \text { in } \Omega,\left.\eta\right|_{\partial \Omega}=0,|\nabla \eta(x)|>0 \text { in } \overline{\Omega \backslash \omega_{0}} .
$$

Let $\Omega:=\{x ;|x|<1\}$ and $\omega_{0}:=\left\{x ;|x|<\frac{1}{2}\right\}$. Then $\eta(x)=1-|x|^{2}$ satisfies (2.1). Set

$$
\varphi(x, t)=\frac{e^{\lambda \eta}}{t(T-t)}, \alpha(x, t)=\frac{e^{\lambda \eta(x)}-e^{2 \lambda\|\eta\|_{C(\bar{\Omega})}}}{t(T-t)},
$$

where $\lambda>0$, then we have the following Carleman estimates with two large parameters which were proved in [12].

Lemma 2.2. Let

$$
\left\{\begin{array}{l}
\partial_{t} y-a(x, t) \Delta y+b(x, t) \cdot \nabla y+c(x, t) y=g \text { in } Q:=\Omega \times(0, T) \\
y=0 \text { on } \partial \Omega \times(0, T)
\end{array}\right.
$$

where $\|a\|_{C^{1}(\bar{Q})}+\|b\|_{L^{\infty}(Q)}+\|c\|_{L^{\infty}(Q)} \leq M, g \in L^{2}(Q)$. Then there exists a number $\lambda_{0}>0$ such that for an arbitrary $\lambda \geq \lambda_{0}$, we can choose a constant $s_{0}(\lambda) \geq 0$ satisfying: there exist a constant $c:=c\left(s_{0}, \lambda_{0}\right)>0$ such that

$$
\begin{aligned}
& \int_{Q}\left\{(s \varphi)^{p-1}\left(\left|\partial_{t} y\right|^{2}+\sum_{i, j=1}^{n}\left|\partial_{i} \partial_{j} y\right|^{2}\right)\right. \\
& \left.\quad+(s \varphi)^{p+1} \lambda^{2}|\nabla y|^{2}+(s \varphi)^{p+3} \lambda^{4} y^{2}\right\} e^{2 s \alpha} d x d t \\
& \quad \leq c \int_{Q}(s \varphi)^{p}|g|^{2} e^{2 s \alpha} d x d t+c \int_{\omega \times(0, T)}(s \varphi)^{p+3} \lambda^{4} y^{2} e^{2 s \alpha} d x d t,
\end{aligned}
$$

for all $s>s_{0}, p=0,1,2$. 


\section{Proof of Theorem 1.1}

First we assume that $t_{0}=T / 2$ without loss of generality by changing the scale of $\mathrm{t}$. Applying Lemma $2.2(p=2)$ to the solution $u$ of (1.1), we have the estimate

$$
\begin{aligned}
& \int_{Q}\left\{s \varphi\left(\left|\partial_{t} u\right|^{2}+\sum_{i, j=1}^{n}\left|\partial_{i} \partial_{j} u\right|^{2}\right)+(s \varphi)^{3} \lambda^{2}|\nabla u|^{2}+(s \varphi)^{5} \lambda^{4} u^{2}\right\} e^{2 s \alpha} d x d t \\
& \leq c \int_{Q}(s \varphi)^{2}\left(f_{1}^{2}+|\nabla \nu|^{2}+|v|^{2}\right) e^{2 s \alpha} d x d t+c \int_{\omega \times(0, T)}(s \varphi)^{5} \lambda^{4} u^{2} e^{2 s \alpha} d x d t
\end{aligned}
$$

Similarly, applying Lemma $2.2(p=1)$ to the solution $v$ of $(1.1)$, we get

$$
\begin{aligned}
& \int_{Q}\left\{\left|\partial_{t} v\right|^{2}+\sum_{i, j=1}^{n}\left|\partial_{i} \partial_{j} v\right|^{2}+(s \varphi)^{2} \lambda^{2}|\nabla v|^{2}+(s \varphi)^{4} \lambda^{4} v^{2}\right\} e^{2 s \alpha} d x d t \\
& \leq c \int_{Q} s \varphi\left(f_{2}^{2}+|\Delta u|^{2}+|\nabla u|^{2}+|u|^{2}\right) e^{2 s \alpha} d x d t \\
& \quad+c \int_{\omega \times(0, T)}(s \varphi)^{4} \lambda^{4} v^{2} e^{2 s \alpha} d x d t .
\end{aligned}
$$

By $\varphi \geq \frac{1}{t(T-t)} \geq \frac{4}{T^{2}},(2 c) \times(3.1)+(3.2)$ and $\lambda$ sufficiently large, we deduce that

$$
\begin{aligned}
& \int_{Q}\left\{s \varphi\left(\left|\partial_{t} u\right|^{2}+\sum_{i, j=1}^{n}\left|\partial_{i} \partial_{j} u\right|^{2}\right)+(s \varphi)^{3} \lambda^{2}|\nabla u|^{2}+(s \varphi)^{5} \lambda^{4} u^{2}\right. \\
& \left.\quad+\left(\left|\partial_{t} v\right|^{2}+\sum_{i, j=1}^{n}\left|\partial_{i} \partial_{j} v\right|^{2}\right)+(s \varphi)^{2} \lambda^{2}|\nabla v|^{2}+(s \varphi)^{4} \lambda^{4} v^{2}\right\} e^{2 s \alpha} d x d t \\
& \leq c \int_{Q}\left[(s \varphi)^{2} f_{1}^{2}+s \varphi f_{2}^{2}\right] e^{2 s \alpha} d x d t+\widetilde{c} \int_{\omega \times(0, T)}\left(u^{2}+v^{2}\right) d x d t .
\end{aligned}
$$

Taking $\partial_{t}$ to the first equation of (1.1), we see that

$$
\partial_{t} u_{t}=\mu_{1} \Delta u_{t}+\partial_{t} \mu_{1} \Delta u+\partial_{t} \ell_{1}+\partial_{t} f_{1} .
$$

Applying Lemma $2.2(p=1)$ to (3.4), we have

$$
\begin{aligned}
& \int_{Q}\left\{\left|\partial_{t}^{2} u\right|^{2}+\sum_{i, j=1}^{n}\left|\partial_{i} \partial_{j} u_{t}\right|^{2}+(s \varphi)^{2} \lambda^{2}\left|\nabla u_{t}\right|^{2}+(s \varphi)^{4} \lambda^{4} u_{t}^{2}\right\} e^{2 s \alpha} d x d t \\
& \leq c \int_{Q} s \varphi\left(\left|\partial_{t} f_{1}\right|^{2}+|\Delta u|^{2}+|\nabla u|^{2}+|u|^{2}+|v|^{2}+|\nabla v|^{2}\right. \\
& \left.\quad+\left|v_{t}\right|^{2}+\left|\nabla v_{t}\right|^{2}\right) e^{2 s \alpha} d x d t+\widetilde{c} \int_{\omega \times(0, T)}\left|u_{t}\right|^{2} d x d t .
\end{aligned}
$$

Taking $\partial_{t}$ to the second equation of (1.1), we find that

$$
\partial_{t} v_{t}=\mu_{0} \Delta u_{t}+\mu_{2} \Delta v_{t}+\partial_{t} \mu_{0} \Delta u+\partial_{t} \mu_{2} \Delta v+\partial_{t} \ell_{2}+\partial_{t} f_{2} .
$$


Applying Lemma $2.2(p=0)$ to $(3.6)$, we have

$$
\begin{gathered}
\left.\int_{Q}\left\{\frac{1}{s \varphi}\left(\left|\partial_{t}^{2} v\right|^{2}+\sum_{i, j=1}^{n}\left|\partial_{i} \partial_{j} v_{t}\right|^{2}\right)+s \varphi \lambda^{2}\left|\nabla v_{t}\right|^{2}+(s \varphi)^{3} \lambda^{4} v_{t}^{2}\right)\right\} e^{2 s \alpha} d x d t \\
\leq c \int_{Q}\left(\left|\partial_{t} f_{2}\right|^{2}+\left|\Delta u_{t}\right|^{2}+\left|\nabla u_{t}\right|^{2}+\left|u_{t}\right|^{2}+|\Delta u|^{2}+|\nabla u|^{2}+|u|^{2}\right. \\
\left.\quad+|\Delta v|^{2}+|\nabla v|^{2}+|v|^{2}\right) e^{2 s \alpha} d x d t+\widetilde{c} \int_{\omega \times(0, T)}\left|v_{t}\right|^{2} d x d t .
\end{gathered}
$$

Using $(2 c) \times(3.5)+(3.7), \phi \geq \frac{4}{T^{2}}$ and $\lambda$ sufficiently large, we find that

$$
\begin{aligned}
\int_{Q}\left\{\left|\partial_{t}^{2} u\right|^{2}+\sum_{i, j=1}^{n}\left|\partial_{i} \partial_{j} u_{t}\right|^{2}+(s \varphi)^{2} \lambda^{2}\left|\nabla u_{t}\right|^{2}+(s \varphi)^{4} \lambda^{4} u_{t}^{2}\right. \\
\left.\quad+\frac{1}{s \varphi}\left(\left|\partial_{t}^{2} v\right|^{2}+\sum_{i, j=1}^{n}\left|\partial_{i} \partial_{j} v_{t}\right|^{2}\right)+s \varphi \lambda^{2}\left|\nabla v_{t}\right|^{2}+(s \varphi)^{3} \lambda^{4} v_{t}^{2}\right\} e^{2 s \alpha} d x d t \\
\leq c \int_{Q}\left[s \varphi\left|\partial_{t} f_{1}\right|^{2}+\left|\partial_{t} f_{2}\right|^{2}+s \varphi\left(|\Delta u|^{2}+|\nabla u|^{2}+|u|^{2}+|v|^{2}\right.\right. \\
\left.\left.\quad+|\nabla v|^{2}\right)+|\Delta v|^{2}\right] e^{2 s \alpha} d x d t+\widetilde{c} \int_{\omega \times(0, T)}\left(\left|u_{t}\right|^{2}+\left|v_{t}\right|^{2}\right) d x d t .
\end{aligned}
$$

Combining (3.3), (3.8), $\phi \geq \frac{4}{T^{2}}$ and $s$ sufficiently large, we obtain

$$
\begin{aligned}
I:= & \int_{Q}\left\{\left|\partial_{t}^{2} u\right|^{2}+\sum_{i, j=1}^{n}\left|\partial_{i} \partial_{j} u_{t}\right|^{2}+(s \varphi)^{2} \lambda^{2}\left|\nabla u_{t}\right|^{2}+(s \varphi)^{4} \lambda^{4} u_{t}^{2}\right. \\
& +s \varphi \sum_{i, j=1}^{n}\left|\partial_{i} \partial_{j} u\right|^{2}+(s \varphi)^{3} \lambda^{2}|\nabla u|^{2}+(s \varphi)^{5} \lambda^{4} u^{2} \\
& +\frac{1}{s \varphi}\left(\left|\partial_{t}^{2} v\right|^{2}+\sum_{i, j=1}^{n}\left|\partial_{i} \partial_{j} v_{t}\right|^{2}\right)+s \varphi \lambda^{2}\left|\nabla v_{t}\right|^{2}+(s \varphi)^{3} \lambda^{4} v_{t}^{2} \\
& \left.+\sum_{i, j=1}^{n}\left|\partial_{i} \partial_{j} v\right|^{2}+(s \varphi)^{2} \lambda^{2}|\nabla v|^{2}+(s \varphi)^{4} \lambda^{4} v^{2}\right\} e^{2 s \alpha} d x d t \\
\leq & c \int_{Q}\left[(s \varphi)^{2} f_{1}^{2}+s \varphi f_{2}^{2}+s \varphi\left(\partial_{t} f_{1}\right)^{2}+\left(\partial_{t} f_{2}\right)^{2}\right] e^{2 s \alpha} d x d t \\
& +\widetilde{c} \int_{\omega \times(0, T)}\left(u^{2}+u_{t}^{2}+v^{2}+v_{t}^{2}\right) d x d t \\
\leq & c \int_{Q}\left[(s \varphi)^{2} f_{1}^{2}(x, T / 2)+s \varphi f_{2}^{2}(x, T / 2)\right] e^{2 s \alpha} d x d t \\
& +\widetilde{c} \int_{\omega \times(0, T)}\left(u^{2}+u_{t}^{2}+v^{2}+v_{t}^{2}\right) d x d t .
\end{aligned}
$$

On the other hand, in terms of $u_{t}^{2}(x, 0) e^{2 s \alpha(x, 0)}=0, v_{t}^{2}(x, 0) e^{2 s \alpha(x, 0)}=0,\left|\alpha_{t}\right| \leq c \varphi^{2}$, and equations (1.1) (1.2) at time $t=T / 2$, we obtain

$$
\int_{\Omega}\left[s^{2} f_{1}^{2}(x, T / 2)+s f_{2}^{2}(x, T / 2)\right] e^{2 s \alpha(x, T / 2)} d x
$$




$$
\begin{aligned}
\leq & c \int_{\Omega}\left(s^{2}\left|\partial_{t} u(x, T / 2)\right|^{2}+s\left|\partial_{t} v(x, T / 2)\right|^{2}\right) e^{2 s \alpha(x, T / 2)} d x \\
& +c\|u(\cdot, T / 2)\|_{H^{2}}^{2}+c\|v(\cdot, T / 2)\|_{H^{2}}^{2} \\
= & c \int_{0}^{\frac{T}{2}} \frac{\partial}{\partial_{t}} \int_{\Omega}\left[s^{2} u_{t}^{2}(x, t)+s v_{t}^{2}(x, t)\right] e^{2 s \alpha(x, t)} d x \\
& +c\|u(\cdot, T / 2)\|_{H^{2}}^{2}+c\|v(\cdot, T / 2)\|_{H^{2}}^{2} \\
\leq & \int_{0}^{\frac{T}{2}} \int_{\Omega}\left(2 s^{3} \partial_{t} \alpha u_{t}^{2}+2 s^{2} u_{t} u_{t t}+2 s^{2} \partial_{t} \alpha v_{t}^{2}+2 s v_{t} v_{t t}\right) e^{2 s \alpha} d x d t \\
& +c\|u(\cdot, T / 2)\|_{H^{2}}^{2}+c\|v(\cdot, T / 2)\|_{H^{2}}^{2} \\
\leq & \int_{Q}\left(2 s^{3} \varphi^{2} u_{t}^{2}+2 s^{2} u_{t} u_{t t}+2 s^{2} \varphi^{2} v_{t}^{2}+2 s v_{t} v_{t t}\right) e^{2 s \alpha} d x d t \\
& +c\|u(\cdot, T / 2)\|_{H^{2}}^{2}+c\|v(\cdot, T / 2)\|_{H^{2}}^{2} \\
\leq & c I+c\|u(\cdot, T / 2)\|_{H^{2}}^{2}+c\|v(\cdot, T / 2)\|_{H^{2}}^{2} \\
\leq & c \int_{Q}\left[(s \varphi)^{2} f_{1}^{2}(x, T / 2)+s \varphi f_{2}^{2}(x, T / 2)\right] e^{2 s \alpha} d x d t \\
& +c\|u(\cdot, T / 2)\|_{H^{2}}^{2}+c\|v(\cdot, T / 2)\|_{H^{2}}^{2} \\
& +\widetilde{c} \int_{\omega \times(0, T)}\left(u^{2}+u_{t}^{2}+v^{2}+v_{t}^{2}\right) d x d t .
\end{aligned}
$$

By the same calculation as that in [4], we deduce that

$$
\begin{aligned}
& \int_{Q}\left[(s \varphi)^{2} f_{1}^{2}(x, T / 2)+s \varphi f_{2}^{2}(x, T / 2)\right] e^{2 s \alpha} d x d t \\
& \quad \leq \frac{c}{\sqrt{s}} \int_{\Omega}\left[s^{2} f_{1}(x, T / 2)+s f_{2}^{2}(x, T / 2)\right] e^{2 s \alpha(x, T / 2)} d x .
\end{aligned}
$$

Combining (3.10), (3.11) and $s$ sufficiently large, we arrive at

$$
\begin{aligned}
\int_{Q}\left(f_{1}^{2}+f_{2}^{2}\right) d x d t \leq & c \int_{\Omega}\left(s^{2} f_{1}^{2}(x, T / 2)+s f_{2}^{2}(x, T / 2)\right) e^{2 s \alpha(x, T / 2)} d x \\
\leq & c\|u(\cdot, T / 2)\|_{H^{2}}^{2}+c\|v(\cdot, T / 2)\|_{H^{2}}^{2} \\
& +\widetilde{c} \int_{\omega \times(0, T)}\left(u^{2}+u_{t}^{2}+v^{2}+v_{t}^{2}\right) d x d t .
\end{aligned}
$$

Hence we complete the proof.

\section{Appendix}

In this appendix, we prove that there exists a smooth and invertible matrix $P=P(x, t)$ such that

$$
P^{-1} A P=P^{-1}\left(\begin{array}{ll}
a_{11} & a_{12} \\
a_{21} & a_{22}
\end{array}\right) P=\left(\begin{array}{cc}
\mu_{1} & 0 \\
\mu_{0} & \mu_{2}
\end{array}\right),
$$


where $\mu_{1}$ and $\mu_{2}$ are eigenvalues of $A$. If $a_{11}(x, t) \geq a_{22}(x, t)$, we define

$$
\begin{aligned}
& \mu_{1}=\frac{a_{11}+a_{22}+\sqrt{\left(a_{11}+a_{22}\right)^{2}-4\left(a_{11} a_{22}-a_{12} a_{21}\right)}}{2}, \\
& \mu_{2}=\frac{a_{11}+a_{22}-\sqrt{\left(a_{11}+a_{22}\right)^{2}-4\left(a_{11} a_{22}-a_{12} a_{21}\right)}}{2} .
\end{aligned}
$$

If $a_{11}(x, t)<a_{22}(x, t)$, we define

$$
\begin{aligned}
& \mu_{1}=\frac{a_{11}+a_{22}-\sqrt{\left(a_{11}+a_{22}\right)^{2}-4\left(a_{11} a_{22}-a_{12} a_{21}\right)}}{2}, \\
& \mu_{2}=\frac{a_{11}+a_{22}+\sqrt{\left(a_{11}+a_{22}\right)^{2}-4\left(a_{11} a_{22}-a_{12} a_{21}\right)}}{2} .
\end{aligned}
$$

Let

$$
\mu_{0}(x, t)=a_{21}(x, t) \text { and } P=\left(\begin{array}{cc}
1 & \frac{a_{12}}{\mu_{2}-a_{11}} \\
0 & 1
\end{array}\right),
$$

we can easily check that

$$
\left(\begin{array}{ll}
a_{11} & a_{12} \\
a_{21} & a_{22}
\end{array}\right)\left(\begin{array}{cc}
1 & \frac{a_{12}}{\mu_{2}-a_{11}} \\
0 & 1
\end{array}\right)=\left(\begin{array}{cc}
1 & \frac{a_{12}}{\mu_{2}-a_{11}} \\
0 & 1
\end{array}\right)\left(\begin{array}{cc}
\mu_{1} & 0 \\
a_{21} & \mu_{2}
\end{array}\right) .
$$

and

$$
\mu_{2}(x, t)-a_{11}(x, t) \neq 0 .
$$

The proof is complete.

\section{References}

[1] M. Yamamoto, Carleman estimates for parabolic equations and applications, Inverse Problems, 25(2009), no. 12, 123013, (75pp).

[2] A. L. Bukhgeim and M. V. Klibanov, Global uniqueness of a class of multidimensional inverse problems, Soviet Math. Dokl., 24(1981), 244-247.

[3] M. V. Klibanov, Estimates of initial conditions of parabolic equations and inequalities via lateral Cauchy data, Inverse Problems, 22(2006), 495-514.

[4] O. Y. Imanuvilov and M. Yamamoto, Lipschitz stability in inverse parabolic problems by the Carleman estimate, Inverse Problems, 14(1998), 1229-45.

[5] M. Choulli, O. Y. Imanuvilov and M. Yamamoto, Inverse source problem for the Navier-Stokes equations, arXiv:UTMS 2006-3.

[6] J. Fan, Y. Jiang, G. Nakamura, Inverse problems for the Boussinesq system, Inverse Problems, 25(2009), no. 8, 085007, (10pp).

[7] A. Benabdallah, M. Cristofol, P. Gaitan and M. Yamamoto, Inverse problem for a parabolic system with two components by measurements of one component, Appl. Anal., 88(2009), 683-709.

[8] V. Isakov, Inverse Source Problems (Providence, RI: American Mathematical Society), 1990.

[9] V. Isakov, Inverse Problems for Partial Differential Equations 2nd edn (Berlin: Springer), 2006.

[10] D. Chae, O. Y. Imanuvilov and S. M. Kim, Exact controllability for semilinear parabolic equations with Neumann boundary conditions, J. Dyn. Control Syst., 2(1996), 449-83. 
[11] A. V. Fursikov and O. Y. Imanuvilov, Controllability of Evolution Equations (Lecture Notes vol 34) (Seoul, Korea: Seoul National University), 1996.

[12] O. Yu. Imanuilov, Controllability of parabolic equations, Sbornik Math., 186(1995), 879-900.

Department of Applied Mathematics, Nanjing Forestry University, Nanjing 210037, P.R. China.

E-mail: fanjishan@njfu.com.cn

Department of Information Management, National Kaohsiung University of Applied Sciences, Kaohsiung 807, Taiwan.

E-mail: chenkj@kuas.edu.tw 